\title{
Mindfulness training: an adjunctive role in the management of chronic illness?
}

Kaveh Monshat MPsychMed, MB BS, FRANZCP Lecturer in Psychiatry

David J Castle MSc, MD, FRANZCP, Chair of Psychiatry

St Vincent's Mental Health University of Melbourne Melbourne, VIC

k.monshat@ unimelb.edu.au

MJA 2012; 196: 569-571 doi: 10.5694/mjall.10974 on Kabat-Zinn, the most influential figure in bringing mindfulness to health care in recent decades, defines it as "the awareness that emerges through paying attention on purpose, in the present moment, and non-judgmentally to the unfolding of experience moment by moment" ${ }^{1}$ Thoughts, judgements and emotional reactions about experience can seem inseparable from the experience itself. This may be more so when the experience is unpleasant, and can create an additional, unnecessary, layer of distress. For example, a new slight pain in the back may be bearable in and of itself, but the suffering experienced is exaggerated greatly if thoughts about it (eg, as a harbinger of future disability) take hold.

A mindful stance involves noticing when one is caught up in reactions to experience or thoughts about the future or the past, recognising whether these are helpful or not, and developing the ability to disengage from them. The capacity and tendency to be mindful is an inherent part of day-to-day life and tends to grow with maturation. Nonetheless, being caught up in ruminative mental content is a common experience for most people. As such, it can take a significant amount of commitment, practice and guidance to cultivate a mindful stance as the predominant mode of relating to internal and external experience. This process can be enhanced through mindfulness training (MT). ${ }^{2}$

\section{Mindfulness training and physical ill health}

Research and clinical practice involving MT is in a phase of exponential growth - in each successive 5-year period between 1991 and 2010, the numbers of articles with "mindfulness" in the title indexed in MEDLINE were four, 14, 69 and 372, respectively. MT comprises instruction in a variety of practices and associated discussion: from sitting meditation with attention focused on the breath, to detailed awareness of movements (during walking meditation or with stretches), and conducting day-to-day activities mindfully. Various strands of these practices have been formally used as, or influenced development of, therapies in Western medicine, particularly following the exposure of the military medical corps to Eastern healing practices in the aftermath of World War II. ${ }^{3}$ Mindfulness meditation is to be distinguished from purely concentration-focused (eg, "transcendental") meditation, where chants or mantras may be used. Also, although stretching is used as a form of meditation, the overarching philosophy of MT differs from and is less culture-bound than that of yoga. Additionally, while relaxation may occur as a side effect of MT, the primary goal of MT is developing non-judgemental awareness of any inner state, and differs as such from relaxation training.

\begin{abstract}
iummary
Narrowly defined, mindfulness is the tendency to encounter moment-to-moment experience without being lost in unhelpful or distressing thoughts triggered by the experience.

- Mindfulness training involves group instruction in and discussion of a variety of meditation styles aimed at enhancing this tendency in daily life.

- There is an accumulating evidence base, albeit of variable quality, which suggests that mindfulness training, when used as part of an integrated approach to chronic disease management, may help alleviate associated psychological distress and improve patients' quality of life.
\end{abstract}

In the past 50 years, the most compelling body of empirical evidence for psychological treatment has pertained to cognitive behaviour therapy, wherein symptoms (eg, an unfounded thought about future disability) are challenged and actively extinguished. The growing popularity of MT reflects and extends the recognition that detailed awareness and acceptance of symptoms may also have a transformative role. $^{3}$ Awareness allows one to distinguish the relative contribution to unpleasantness of the sensory experience of a symptom (eg, pain) from thoughts about it (eg, "I'm going to become an invalid") or emotions triggered by it (eg, fear). Acceptance is used here in the sense of not creating unnecessary distress and distraction by fighting the fact that something unwanted has happened (eg, "Why me?"). It is not a passivity or "giving up" in the face of difficulties. Patients are often relieved to find that they can gradually let go of their reactions to a symptom and surprised to learn that the symptom itself thus becomes more tolerable. ${ }^{4}$

This approach is particularly relevant when distressing thoughts and associated anxiety and depressive symptoms are continually generated, and may worsen outcomes, in the context of chronic physical disease. ${ }^{5}$ Chronic disease accounts for two-thirds of health care expenditure in Australia. ${ }^{6}$ Reducing associated psychological morbidity may, directly and through enhanced patient self-management, lead to improved health outcomes and reduced health care costs. ${ }^{7}$

The most commonly studied MT program, mindfulness-based stress reduction (MBSR), developed by KabatZinn at the University of Massachusetts Medical Center in 1979, was aimed at chronically ill general medical patients who had exhausted physical treatment possibilities. ${ }^{4}$ The program involves eight weekly 2.5 -hour sessions and a 7-hour weekend session, usually delivered by one facilitator to a group of 10-30 participants. An 
Selected randomised controlled trials of mindfulness-based interventions in patients with chronic physical disorders*

\begin{tabular}{|c|c|c|c|c|}
\hline Study & $\begin{array}{l}\text { Intervention } \\
\text { and control }\end{array}$ & $\begin{array}{l}\text { Sample } \\
\text { size }\end{array}$ & Disorder & Outcome measures and statistically significant results ${ }^{\dagger}$ \\
\hline Astin et $\mathrm{al}^{13}$ & $\begin{array}{l}\text { MBSR + qigong } \\
\text { Education }\end{array}$ & $\begin{array}{l}64 \\
64\end{array}$ & Fibromyalgia & $\begin{array}{l}\text { No between-group difference in improvements at up to } 6 \text { months (SF-36 quality of life and BDI } \\
\text { depression scores) }\end{array}$ \\
\hline Bränström et $\mathrm{al}^{14}$ & $\begin{array}{l}\text { MBSR } \\
\text { Waitlist }\end{array}$ & $\begin{array}{l}32^{\ddagger} \\
39\end{array}$ & Cancer $^{5}$ & $\begin{array}{l}\text { Pre-post improvement in perceived stress (PSS: } \mathrm{ES}=0.63, P=0.004 \text { ) and positive mental state } \\
\text { (PSOM: ES }=0.5, P=0.02 \text { ), which did not remain significant at } 6 \text {-month follow-up }{ }^{15}\end{array}$ \\
\hline Grossman et $\mathrm{al}^{16}$ & $\begin{array}{l}\text { MBSR } \\
\text { Waitlist }\end{array}$ & $\begin{array}{l}76^{\ddagger} \\
74\end{array}$ & $\begin{array}{l}\text { Multiple } \\
\text { sclerosis }\end{array}$ & $\begin{array}{l}\text { Pre-post improvement in quality of life (PQOLC: } \mathrm{ES}=0.86, P<0.001 \text {; HAQUAMS: } \mathrm{ES}=0.43 \text {, } \\
P<0.001 \text { ), and reductions in depressive symptoms }(\mathrm{CES}-\mathrm{D}: \mathrm{ES}=0.65, P<0.001 \text { ), anxiety } \\
\text { (STAl: } \mathrm{ES}=0.39, P<0.001 \text { ) and fatigue (MFIS: } \mathrm{ES}=0.41, P<0.001 \text { ), which remained significant at } \\
\text { 6-month follow-up }\end{array}$ \\
\hline Lengacher et $\mathrm{al}^{17}$ & $\begin{array}{l}\text { MBSR } \\
\text { Waitlist }\end{array}$ & $\begin{array}{l}41^{\ddagger} \\
43\end{array}$ & Breast cancer & $\begin{array}{l}\text { Pre-post reductions in anxiety (STAI state subscale: } \text { mean, } \mathrm{MBSR}=28.3, \mathrm{WL}=33.0, P=0.03 \text {; } \mathrm{STAl} \\
\text { trait subscale: } \text { mean, } \mathrm{MBSR}=30.4, \mathrm{WL}=34.5, P=0.004 \text { ) and symptoms of depression (CES-D: } \\
\text { mean, } \mathrm{MBSR}=6.3, \mathrm{WL}=9.6, P=0.03 \text { ), and improvement in quality of life (SF-36 physical } \\
\text { functioning subscale: } \text { mean, } \mathrm{MBSR}=50.1, \mathrm{WL}=47.0, P=0.01)^{\mathrm{q}}\end{array}$ \\
\hline Monti et $\mathrm{al}^{18}$ & $\begin{array}{l}\text { MBAT } \\
\text { Waitlist }\end{array}$ & $\begin{array}{l}56^{\ddagger} \\
55\end{array}$ & Cancer $^{\zeta}$ & $\begin{array}{l}\text { Pre-post reduction in psychological distress (SCL-90: mean change, MBAT }=-0.20, \mathrm{WL}=-0.04 \text {, } \\
P<0.001 \text { ) and improvement in quality of life (SF-36 mental health subscale: mean change, } \\
\text { MBAT }=13.05, \mathrm{WL}=2.16, P<0.001 \text { ) }\end{array}$ \\
\hline Mularski et al ${ }^{19}$ & $\begin{array}{l}\text { MBBT } \\
\text { Support group }\end{array}$ & $\begin{array}{l}44 \\
42\end{array}$ & COPD & $\begin{array}{l}\text { No pre-post between-group difference in quality of life (SF-36 for Veterans), dyspnoea or rates of } \\
\text { exacerbation }\end{array}$ \\
\hline Pradhan et $\mathrm{al}^{20}$ & $\begin{array}{l}\text { MBSR } \\
\text { Waitlist }\end{array}$ & $\begin{array}{l}31 \\
32\end{array}$ & $\begin{array}{l}\text { Rheumatoid } \\
\text { arthritis }\end{array}$ & $\begin{array}{l}\text { Reduction in psychological distress (SCL-90: mean change, MBSR }=-0.17, \mathrm{WL}=-0.03, P=0.04 \text { ) and } \\
\text { improvement in psychological wellbeing (PWBS: mean change, } \mathrm{MBSR}=5.55, \mathrm{WL}=-5.47, P=0.03 \text { ) } \\
\text { at } 6 \text { months after program commencement (note: MBSR group received three refresher classes in } \\
\text { the } 4 \text { months after completing the 2-month program) }\end{array}$ \\
\hline Speca et $\mathrm{al}^{21}$ & $\begin{array}{l}\text { MBSR } \\
\text { Waitlist }\end{array}$ & $\begin{array}{l}53 \\
37\end{array}$ & Cancer $^{5}$ & $\begin{array}{l}\text { Pre-post reductions in mood disturbance (POMS: } \text { mean change, } \mathrm{MBSR}=-24.1, \mathrm{WL}=-2.1, P<0.001 \text { ), } \\
\text { anxiety (POMS: mean change, } \mathrm{MBSR}=-4.8, \mathrm{WL}=-0.4, P<0.001 \text { ) and stress symptoms (SOSI: } \\
\text { mean change, } \mathrm{MBSR}=-31.3, \mathrm{WL}=-12.3, P<0.01 \text { ) }\end{array}$ \\
\hline Zautra et al $^{22}$ & $\begin{array}{l}\text { MP } \\
\text { CBT for pain } \\
\text { Education }\end{array}$ & $\begin{array}{l}48 \\
52 \\
44\end{array}$ & $\begin{array}{l}\text { Rheumatoid } \\
\text { arthritis }\end{array}$ & $\begin{array}{l}\text { Pre-post improvement in patients with a previous history of recurrent depression compared with } \\
\text { CBT and education groups for clinician-assessed swelling (DAS-28: } \mathrm{ES}=-0.62, P<0.001 \text { ), } \\
\text { tenderness (DAS-28: } \mathrm{ES}=0.83, P<0.001 \text { ), affect (PANAS positive affect subscale: } \mathrm{ES}=0.78 \text {, } \\
P<0.001 \text {; negative affect subscale: } \mathrm{ES}=-0.89, P<0.01 \text { ) and relationship with pain (CSQ pain } \\
\text { catastrophising: } \mathrm{ES}=-0.18, P<0.001 \text {; pain coping: } \mathrm{ES}=0.65, P<0.05 \text { ) }\end{array}$ \\
\hline
\end{tabular}

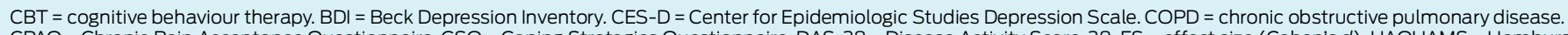

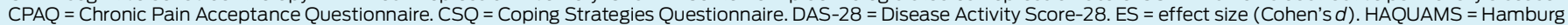

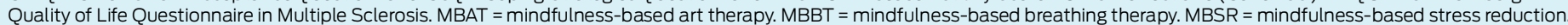
MFIS = Modified Fatigue Impact Scale. MP = mindfulness program based on MBSR designed specifically for patient group. PANAS = Positive And Negative Affect Schedule. POMS = Profile of Mood States. PQOLC = Profile of Health-Related Quality of Life in Chronic Disorders. PSOM = Positive States of Mind scale. PSS = Perceived Stress Scale.

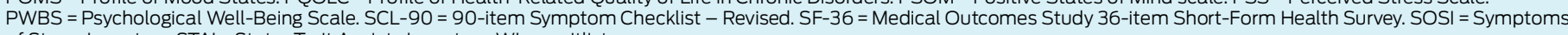
of Stress Inventory. STAI = State-Trait Anxiety Inventory. WL = waitlist.

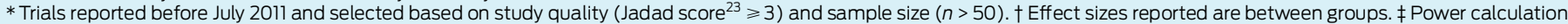

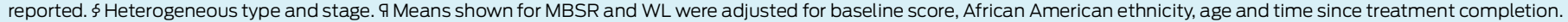

educational, rather than therapeutic, approach is used, and modules include the neurophysiology of stress and exercises aimed at enhancing how stressful life situations may be dealt with. There is a requirement for home practice of various forms of meditation and other exercises for 45-60 minutes per day.

Unless delivered in an extended silent retreat - and undertaken by an individual with a fragile mental state MT does not appear to be associated with adverse events. ${ }^{2}$ Nonetheless, patients with active psychotic symptoms, substance dependence or significant cognitive impairment are generally excluded from MT group programs, unless the programs are designed specifically for such populations. ${ }^{2}$

\section{Accumulating evidence of variable quality}

Several recent systematic reviews and meta-analyses have collated the evidence pertaining to MT in healthy participants, patients with psychiatric disorders, and those with a physical illness. ${ }^{8-12}$ Here, we focus on studies involving general medical patients - randomised controlled trials (RCTs) of relatively high quality and large sample size are summarised in the Box. While MT research in Australia has largely focused on psychiatric populations, studies in general medical patients are also beginning to emerge (eg, patients with cancer ${ }^{24,25}$ and chronic back pain ${ }^{26}$ ).

\section{Summary of the evidence}

A recent meta-analysis of eight RCTs $(n=667)$ of MBSR for patients suffering chronic medical illness included seven studies with a waitlist group as a control; the other one used an education/support program. ${ }^{9}$ All but one study reported attrition rates below 25\%. Six studies met Cochrane criteria for medium to high quality. Betweengroup effect sizes were reported using Cohen's $d$, which reflects the difference between means divided by the standard deviation: a value of 0.2 is considered a small positive impact of intervention, 0.5 a medium impact, and $>0.8$ a large impact. The meta-analysis found betweengroup effect sizes of 0.26 (95\% CI, 0.18-0.34; $P<0.001)$ for measures of depression and 0.32 (95\% CI, 0.13-0.50; $P<0.001)$ for overall psychological distress, both without heterogeneity $\left(\mathrm{I}^{2}=0\right)$, and 0.47 (95\% CI, 0.11-0.83; $P<0.05)$ for measures of anxiety, but with considerable heterogeneity $\left(\mathrm{I}^{2}=53.95\right)$.

A meta-analysis of 10 studies $(n=583)$ of MBSR for patients with cancer included only four controlled trials. ${ }^{8}$ Six studies included patients with various cancer types in MT groups, while in four all patients had the same malignancy. Breast cancer was the most common type of cancer across all studies. The mean attrition rate was $23 \%$. The meta-analysis found a within-group effect size $(d)$ after the intervention of $0.48(95 \% \mathrm{CI}, 0.38-0.59$; two-tailed 
$P<0.0001)$ with adequate homogeneity $\left(\chi^{2}=-13.34, \mathrm{df}=6\right)$ for mental health measures, and 0.18 (95\% CI, 0.08-0.28; two-tailed $P<0.0003)$ with poor homogeneity $\left(\chi^{2}=28.72\right.$, $\mathrm{df}=7$ ) for physical health measures.

A recent systematic review of controlled studies of MT for patients with chronic pain included 10 studies $(n=951)$, four of which were RCTs. ${ }^{10}$ Four studies included an active control (cognitive behaviour therapy, progressive muscle relaxation, massage and group support). MT appeared to confer a non-specific (ie, possibly related to factors such as the shared group experience) beneficial effect on measures of pain and depression and a specific effect on pain acceptance or tolerance, day-to-day stress and quality of life. Of note was the persistence of some beneficial effects during follow-up of up to 3 years.

\section{Limitations and challenges}

Many trials in MT have attracted significant criticism ${ }^{10,27,28}$ on methodological grounds such as small sample size, lack of a control group, use of non-intervention control groups (thus disallowing detection of a specific effect from mindfulness), inadequate follow-up, use of non-standardised measures, absence of measures of mindfulness, and a lack or inadequate reporting of randomisation and/or blinding. In addition, differences between exactly how MT was delivered (where this was reported - for example, session length or program duration), heterogeneity of patient populations between (and sometimes within) studies, and variability of effects within the same condition make meaningful comparison between trials difficult. ${ }^{27,29}$

As yet, no compelling evidence exists regarding the costeffectiveness of MT. MBSR, as a particularly resourceintensive example, stipulates 27 hours of contact time with patients and requires highly trained facilitators with their own personal mindfulness practice of several years' duration. This is somewhat mitigated by the fact that groups of up to 30 participants may be conducted by one facilitator. One study reported the encouraging finding that the number of medical visits by patients suffering chronic physical illness was reduced by $27 \%$ in the year after an MBSR program $(P=0.03) .{ }^{30}$

The subjective experience of mindfulness is often complex and somewhat paradoxical (eg, accepting pain while taking reasonable steps to reduce it). Facilitators are required to teach the group based on their own experience of moment-to-moment awareness, making "manualising" interventions and analyses of fidelity difficult. ${ }^{1}$ These factors, among others, have been cited as challenges to quantitative research in MT and suggest the need for including rigorous qualitative assessment as part of the evaluation process. ${ }^{1,31}$ Perhaps not surprisingly, there are conflicting data about the relationship between aspects of MT (duration of training sessions, ${ }^{32}$ engagement with homework practice, ${ }^{29,33}$ and overall amount of meditation experience ${ }^{34}$ ) and the magnitude of effects.

\section{Conclusions}

Chronic disease places a tremendous burden on society that is exacerbated by associated psychological distress. MT holds promise for alleviating such distress and improving patients' quality of life. It should be welcomed with caution, however, considering the methodological issues currently affecting the evidence base. Adequately powered, high-quality clinical trials, ideally including rigorous qualitative evaluation, may help to determine the place of mindfulness as part of an integrated approach to chronic disease management.

Competing interests: We are investigators on a National Health and Medical Research Council grant submission pertaining to mindfulness training.

Provenance: Not commissioned; externally peer reviewed.

1 Kabat-Zinn J. Mindfulness-based interventions in context: past, present, and future. Clin Psychol (New York) 2003; 10 : 144-156. doi: 10.1093/clipsy.bpg016.

2 MaceC. Mindfulness and mental health: therapy, theory and science. London: Routledge, 2007.

3 Dryden W, Still A. Historical aspects of mindfulness and self-acceptance in psychotherapy. J Ration Emot Cogn Behav Ther 2006; 24:3-28. doi: 10.1007/s10942-006-0026-1.

4 Kabat-Zinn J. Full catastrophe living: using the wisdom of your body and mind to face stress, pain, and illness. New York: Random House, 2005.

5 Merkes M. Mindfulness-based stress reduction for people with chronic diseases. Aust J Prim Health 2010; 16: 200-210.

6 DowrickC. The chronic disease strategy for Australia [editorial]. Med J Aust 2006; 185: 61-62.

7 Glasgow NJ, Jeon YH, Kraus SG, Pearce-Brown CL. Chronic disease self-management support: the way forward for Australia. Med J Aust 2008; 189 (10 Suppl): S14-S16.

8 Ledesma D, Kumano H. Mindfulness-based stress reduction and cancer: a meta-analysis. Psychooncology 2009; 18 : 571-579.

9 Bohlmeijer E, Prenger R, Taal E, et al. The effects of mindfulness-based stress reduction therapy on mental health of adults with a chronic medical disease: a meta-analysis. J Psychosom Res 2010; 68: 539-544.

10 Chiesa A, Serretti A. Mindfulness-based interventions for chronic pain: a systematic review of the evidence. J Altern Complement Med 2011; 17: 83-93.

11 Chiesa A, Serretti A. A systematic review of neurobiological and clinical features of mindfulness meditations. Psychol Med 2010; 40:1239-1252.

12 Hofmann SG, Sawyer AT, Witt AA, OhD. The effect of mindfulness-based therapy on anxiety and depression: a metaanalytic review. J Consult Clin Psychol 2010; 78: 169-183.

13 Astin JA, Berman BM, Bausell B, et al. The efficacy of mindfulness meditation plus Qigong movement therapy in the treatment of fibromyalgia: a randomized controlled trial. J Rheumatol 2003; 30: 2257-2262.

14 Bränström R, Kvillemo P, Brandberg Y, Moskowitz JT. Self-report mindfulness as a mediator of psychological well-being in astress reduction intervention for cancer patients--a randomized study. Ann Behav Med 2010; 39: 151-161.

15 Bränström R, Kvillemo P, Moskowitz JT. A randomized study of the effects of mindfulness training on psychological well-being and symptoms of stress in patients treated for cancer at 6-month follow-up. Int J Behav Med 2011; 20 Sep. [Epub ahead of print.]

16 Grossman P, Kappos L, Gensicke H, et al. MS quality of life, depression, and fatigue improve after mindfulness training: a randomized trial. Neurology 2010; 75: 1141-1149.

17 Lengacher CA, Johnson-Mallard V, Post-White J, et al. Randomized controlled trial of mindfulness-based stress reduction (MBSR) for survivors of breast cancer. Psychooncology 2009; 18: 1261-1272.

18 Monti DA, PetersonC, KunkelEJ, et al. A randomized, controlled trial of mindfulness-based art therapy (MBAT) for women with cancer. Psychooncology 2006; 15: 363-373.

19 Mularski RA, Munjas BA, Lorenz KA, et al. Randomized controlled trial of mindfulness-based therapy for dyspnea in chronic obstructive lung disease. J Altern Complement Med 2009; 15: 1083-1090.

20 Pradhan EK, Baumgarten M, Langenberg P, et al. Effect of mindfulness-based stress reduction in rheumatoid arthritis patients. Arthritis Rheum 2007; 57:1134-1142.

21 Speca M, Carlson LE, Goodey E, Angen M. A randomized, wait-list controlled clinical trial: the effect of a mindfulness meditation-based stress reduction program on mood and symptoms of stress in cancer outpatients. Psychosom Med 2000; 62: 613-622.

22 Zautra AJ, Davis MC, Reich JW, et al. Comparison of cognitive behavioral and mindfulness meditation interventions on adaptation to rheumatoid arthritis for patients with and without history of recurrent depression. $J$ Consult Clin Psychol 2008; 76: 408-421.

23 Jadad AR, Moore RA, Carroll D, et al. Assessing the quality of reports of randomized clinical trials: is blinding necessary? Control Clin Trials 1996; 17:1-12.

24 Sharplin GR, Jones SBW, HancockB, et al. Mindfulness-based cognitive therapy: an efficacious community-based group intervention for depression and anxiety in a sample of cancer patients. Med J Aust 2010; 193 (5 Suppl): S79-S82.

25 Foley E, Baillie A, Huxter M, et al. Mindfulness-based cognitive therapy for individuals whose lives have been affected by cancer: a randomized controlled trial. J Consult Clin Psychol 2010; 78: 72-79.

26 Schütze R, Rees C, Preece M, Schütze M. Low mindfulness predicts pain catastrophizing in a fear-avoidance model of chronic pain. Pain 2010; 148: 120-127.

27 Nyklicek I, van Son J, Pouwer F. Two recently published meta-analyses on the effectiveness of mindfulness-based interventions: what should the reader make of it? J Psychosom Res 2010; 69: 613-614.

28 Bishop SR. What do we really know about mindfulness-based stress reduction? Psychosom Med 2002; 64: 71-83.

29 Rosenzweig S, Greeson JM, Reibel DK, et al. Mindfulness-based stress reduction for chronic pain conditions: variation in treatment outcomes and role of home meditation practice.J Psychosom Res 2010; 68: 29-36.

30 Roth B, Stanley TW. Mindfulness-based stress reduction and healthcare utilization in the inner city: preliminary findings. Altern Ther Health Med 2002; 8: 60-66.

31 Grossman P. On measuring mindfulness in psychosomatic and psychological research. J Psychosom Res 2008; 64: 405-408.

32 Carmody J, Baer RA. How long does a mindfulness-based stress reduction program need to be? A review of class contact hours and effect sizes for psychological distress. J Clin Psychol 2009; 65: 627-638.

33 Carmody J, Baer RA. Relationships between mindfulness practice and levels of mindfulness, medical and psychological symptoms and well-being in a mindfulness-based stress reduction program. J Behav Med 2008; 31: 23-33.

34 Buhle J, Wager TD. Does meditation training lead to enduring changes in the anticipation and experience of pain? Pain 2010; 150: 382-383. 